\title{
VI. INTERNATIONAL LEIBNIZ KONGRESS \\ HANOVRE, ALLEMAGNE, 18-23 JUILLET 1994
}

Au cour du Congrès se tiendra la question portant sur la position historique de Leibniz et sur sa signification à cette époque. Depuis le dernier Congrès un nombre important de textes inédits a vu le jour. D'autres publications dues à de nouveaux développements philosophiques invitent à une nouvelle interprétation des textes connus. Le Congrès doit assembler et confronter ces diverses investigations de manière à en déterminer l'intérêt pour la recherche.

Le thème "Leibniz et l'Europe" constituera le point saillant du Congrès. Durant ses voyages en France, en Angleterre, aux Pays-Bas, à Vienne et en Italie, Leibniz a connu personnellement de nombreux savants qui figurent parmi les 1100 interlocuteurs qui resteront en correspondance avec lui. Ses nombreuses œuvres philosophiques sont composées dans une langue française très relevée de la Cour et de la Diplomatie, la plus grande partie de ses textes philosophiques et scientifiques est écrite dans le néo-latin qui était la langue internationale des savants de son siècle. Il lui arrive aussi d'écrire en italien et de lire l'anglais. Dans sa correspondance, dans nombre d'autres écrits, se trouvent mis en évidence d'une manière remarquable ses contacts personnels et épistolaires avec les têtes pensantes les plus significatives des sciences et de la politique en Europe, sans oublier, dans ce contexte européen, ses relations avec la Chine. Ce qui importe, ce ne sont pas seulement ces contacts savants, mais ses efforts théoriques et pratiques pour faire régner la paix entre les États, les courants philosophiques et les confessions religieuses, selon un point de vue très européen. Avec la reconnaissance et l'instauration de la différence dans l'unité à construire, Leibniz développe un concept destiné à modifier la réalité d'alors, dont l'actualisation restait à l'épreuve. Ce sera un but pour le Congrès que d'analyser les relations entre ses efforts politiques et juridiques, aussi bien que ses activités dans la République des Lettres d'une part, et, d'autre part, ses réflexions philosophiques à ce sujet. On s'interrogera aussi sur ce caractère international des théories leibniziennes. L'Europe fut pour Leibniz, au premier chef, une unité culturelle et savante, si bien qu'il a pu désigner la Chine comme une « Europe de L'Est ».

Le Congrès se tiendra à l'Université d'Hanovre. Il s'ouvrira le lundi 18 juillet 1994 à $10 \mathrm{~h}$ avec les discours officiels et une conférence introductive. Le lundi 18 juillet, de 15 à $19 \mathrm{~h}$, et le vendredi 22 juillet, de 15 à $18 \mathrm{~h}$. Des séances plénières sont prevvues. Les sections du Congrès se tiendront le reste du temps. Le mercredi à $20 \mathrm{~h}$ aura lieu une conférence publique. Durant le Congrès, on aura la possibilité de visiter les archives leibniziennes à la Landesbibliothek. Le samedi 23 est prévue une excursion dans les cités leibniziennes de la région.

La Leibniz-Gesellschaft invite tous les chercheurs, spécialistes et amis à prendre part à ce Congrès. Nous recevrons les inscriptions pour les communications de section jusqu'au 15 décembre 1993.

Les demandes d'inscription sont à envoyer à la Gottfried-Wilhelm-LeibnizGesellschaft e. V., Niedersächsische Landesbibliothek, Waterloostr. 8, D-3000 Hannover 1, Telefon 0511/1267-331; Fax : 0511/1267-202. 